1951, calcium was being advocated as suitable therapy for tuberculosis. To determine whether there may be an association between tuberculosis and calcium deficiency, serum calcium, 25- $\mathrm{OHD}_{3}$, parathyroid hormone and other related parameters have been measured in tuberculous patients, and compared with levels in a control population matched for age, sex, and ethnic origin. Tuberculous patients showed a statistically significant deficiency of calcium (uncorrected for albumin) and albumin, but not of $25-\mathrm{OHD}_{3}$, parathyroid hormone or corrected calcium. Alkaline phosphatase and gammaglutamyl transferase were also raised in the patient group.

\section{Pooter's lung}

RB DOUglas Mosquitoes and their debris are usually removed from cages by sucking them into a pipette by mouth. This technique is known as "pooting" and is used by entomologists in field studies to collect sandflies from crevices in walls. Twelve laboratory workers were divided at random into two groups and then matched as closely as possible for age, height, and sex. One group (the "subjects") was asked to poot mosquitoes freely as necessary throughout the course of a working day. The other group (the "controls") carried out other duties in the same laboratory but refrained from pooting insects. Respiratory function was assessed before and after shift by making five blows into a dry wedge spirometer and also a flow-volume system using an integrating pneumotachograph. The results showed that five out of six subjects showed a reduction in forced expiratory volume $\left(\mathrm{FEV}_{1}\right)$ and a somewhat larger fall in forced vital capacity (FVC) over the seven hour shift. Two of the controls had hay-

\section{Correspondence}

\section{Hard metal lung disease}

Sir,-In a paper which was published in Thorax in September 1980 (Thorax 1980;35:653-9), we described four patients with hard metal lung disease. Among other investigations, the amount of some metals in mediastinal lymph nodes had been measured. Since then, one of the patients (case 2) has died from cardiac infarction. Necropsy confirmed pulmonary fibrosis of moderate degree. Pieces of the lung and mediastinal lymph nodes were investigated as to their metal content, and the results can be seen in the table. Within brackets are the earlier values from the nodes taken at mediastinoscopy.

As can be seen, the values in the lung are much lower than those in the lymph nodes. Thus, there is an efficient clearance from the lung via the lymphatic system, and lung biopsies will therefore only yield low values which would be difficult to evaluate. If exposure needs to be ascertained mediastinal lymph nodes should be excised. fever and they also showed a drop in $\mathrm{FEV}_{1}$ and FVC. The other four controls all showed improvement. These results, taken together with a very few complaints of respiratory symptoms (including one man aged 28 years who reported a very severe response to pooting sandflies in France), indicate that the practice may carry a risk of respiratory sensitisation.

\section{Primary seminoma of the thymus}

ER TOWNSEND, RA ISWORTH, HM BRADMORE, B GODWIN, IKR MCMILLAN A review of the literature shows 74 cases of seminomas in the mediastinum have been reported but only 14 of these have been proved to originate from the thymus gland. We report a further three cases. These tumours occur in young males with an average age of 26 years. In our cases the mean age was 21 years. One presented with vague chest pain, another with a sternal swelling and the third was found on a routine chest radiograph. In two cases the tumour was totally removed with no recurrence, but in the third the patient was treated with DXT, and an osteolytic secondary developing 11 months later was successfully treated with DXT. To date all patients remain well and apparently free from recurrence. No evidence of a testicular lesion was found in any of these. The histology in all cases was indistinguishable from a primary testicular seminoma, recognisable thymic tumour was present in all cases, but in one case teratoid elements were present. In these very rare tumours surgical excision is usually possible but like their testicular counterparts they appear to respond well to DXT if excision is incomplete.

Various lymph nodes seem to give slightly different values but this is to be expected since they will take care of other reactions as well and an even distribution would be strange.

GUNNAR HILLERDAL Institutionen für lungmedicin Akademiska sjukhuset, 75014 Uppsala Sweden

Table Amount of metals (ug/g dry tissue)

\begin{tabular}{llc}
\hline & Cobalt & Tungsten \\
\hline Lung parenchyma & & \\
Subpleural upper lobe & $0 \cdot 79$ & $2 \cdot 8$ \\
Subpleural lower lobe & $0 \cdot 14$ & $4 \cdot 9$ \\
Central lower lobe & $0 \cdot 17$ & $6 \cdot 6$ \\
Mediastinal lymph node & $3 \cdot 28(3 \cdot 1)$ & $135 \cdot 0(34 \cdot 0)$ \\
\hline
\end{tabular}

